Usage, Usability, and Utility of 3D City Models, 03009 (2012)

DOI: $10.1051 / 3 \mathrm{u} 3 \mathrm{~d} / 201203009$

(C) Owned by the authors, published by EDP Sciences, 2012

\title{
Children exposure to PM levels in a typical school morning
}

\author{
J. Valente, J.H. Amorim, P. Cascão, V. Rodrigues and C. Borrego \\ CESAM \& Department of Environment and Planning, University of Aveiro, \\ 3810-193 Aveiro, Portugal
}

\begin{abstract}
One of the major challenges to urban sustainability is the threat posed by air pollution, being exposure to ambient air pollutants associated with a high rate of premature deaths. Therefore, the study of the exposure of people, and in particular of vulnerable population groups such as children, to air pollution is a subject of paramount importance. In this paper a CFD model is used to simulate the particulate matter personal exposure of students in their school routine (both daily walk to and permanence in school). Under the concept of COST TU0801, the usability of a 3D city model is evaluated. The analysis was carried out for 4 children, with 4 alternative walking routes to school and using 4 different classrooms. Results indicate that the individual exposure of children is extremely spatially dependent, as a consequence of the wind flow and air pollutant dispersion patterns.
\end{abstract}

\section{INTRODUCTION}

Air pollution is the environmental factor with the highest impact on health in Europe and is responsible for the largest burden of environment-related diseases [1]. Estimates from the European Environment Agency [2] indicate that exposure to particulate matter (PM) causes approximately three million deaths per year globally. Nevertheless, concern should focuses not only on ambient air quality but also on air quality in indoor environments such as homes, workplaces, schools, etc. Indoor air quality appears with a high importance for two main reasons: the time people spend in those environments (more than $90 \%$ ), and the nature and concentration of the pollutants on these spaces, where air contaminants are determined by the artificially made and maintained spaces, dependent on a growing number of new synthetic substances used on the construction, furniture and consumer products [3].

Recent studies have shown that the indoor concentrations of PM derive both from outdoor and indoor sources [4-6]. Nevertheless, each source depends on several variables such as the ventilation rate, outdoor air pollution levels, meteorology, penetration factors, deposition and re-suspension, type of indoor activities, or the size of particles. Therefore, the correct and detailed description of both outdoor and indoor spaces is of paramount importance when studying human exposure to air pollutants. The levels of indoor PM concentrations directly influence the health of their occupants, in particular of vulnerable population subgroups, such as school age children [7,8]. This conclusion stresses the importance of improving our knowledge on the exposure of children, paying particular attention to the school microenvironment.

In order to evaluate the real impacts of air pollution on the population health is then necessary to undergo a complete assessment of human exposure to atmospheric pollutants that includes both outdoor and indoor exposure assessments. Different methodologies can be applied to determine the individual exposure, using direct measurements or estimations based on exposure concentration data and the period of time during which contact occurs. The mathematical basis of human exposure estimation lies down on the microenvironment concept. This is because different microenvironments (e.g. outdoor, inside a

This is an Open Access article distributed under the terms of the Creative Commons Attribution License 2.0, which permits unrestricted use, distribution, and reproduction in any medium, provided the original work is properly cited. 


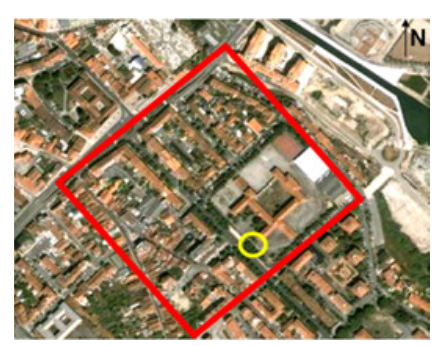

Figure 1. Satellite image (from Google Earth) of the study area (the simulation domain is defined in the red square, and air quality station in yellow circle).

car, inside an office, inside a school) contribute differently to the exposure estimation to atmospheric pollutants, due to both micro environmental concentration ranges and the time fractions spent in each.

Due to the limitations of exposure measurements, in terms of representativeness and cost, exposure modelling, using air quality models is a reliable and useful tool for exposure studies [9]. The use of a computational fluid dynamics (CFD) model is a valuable tool for the detailed understanding of how different human behaviours can affect the exposure and, consequently, the related health effects. Nevertheless, these models still need further research and developments, particularly in terms of indoor spaces modelling. One of the limitations with these models (both for outdoor and indoor spaces) is the description of the geometry and physical characteristics of the domain of simulation.

\section{METHODOLOGY}

PM10 personal exposure of 4 children was calculated for a typical school morning, including the time spent walking to school and the time spent in the classroom. The 3D fields of wind and PM10 concentration were calculated with the CFD model VADIS, while the personal exposure was estimated using MEB model together with indoor/outdoor relationships to take into account exposure in indoor microenvironments. Both numerical tools were developed at the University of Aveiro, Portugal.

\subsection{Study area}

The study domain is a small area at the centre of Aveiro, a medium size town, in Northwest Portugal. The selected study domain is characterized by residential buildings and schools (Figure 1) and it is centred at one of the most important thoroughfares of the town. This area has building blocks of similar height, several secondary roads, and the main Avenue that is flanked by rows of dense tall trees. At the school's front yard there is an air quality station from the national air quality monitoring network. These data were used for validating the air quality results obtained with the model.

Figure 2 shows the 3D geometry, LOD1 typology, created using VADIS model. Since the model uses a structured grid the shape of buildings has to be simplified as regular blocks. Also, neighbouring buildings are assembled based on their configuration and height. Trees are defined as porous blocks, in which the porosity is proportional to the leaf area density.

A brief description of the computational domain and input data is given in Table 1.

\subsubsection{Walking routes and classrooms}

Four alternative pathways to the secondary school, which is centred in the domain, have been selected (Figure 3). To have a time profile for each route we have walked through each pathway carrying a GPS. Instead of using an average walking speed, with this methodology the time spent crossing the roads due 
Table 1. General properties of the computational domain (L: length; W: width; H: height).

\begin{tabular}{|l|l|}
\hline $\mathrm{L} \times \mathrm{W}\left(\mathrm{m}^{2}\right)$ & $380 \times 220$ \\
\hline H/W ratio & 0.75 \\
\hline Mesh resolution and number of cells & $10 \times 10 \times 10,38400$ \\
\hline Buildings: number of blocks and height range $(\mathrm{m})$ & $40,3-15$ \\
\hline Trees: number of blocks, species, height range (m) & $\begin{array}{l}29, \text { Acer pseudoplatanus } \\
\text { and Quercus robur, } 10\end{array}$ \\
\hline Number of roads and width (m) & $12,4-20$ \\
\hline
\end{tabular}

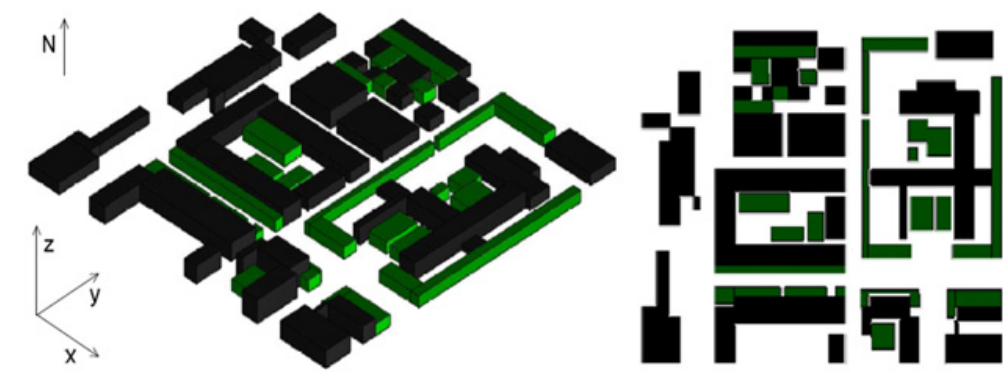

Figure 2. 3D geometry of buildings (in grey) and trees (in green) created by VADIS. (a) 3D view and (b) top view.

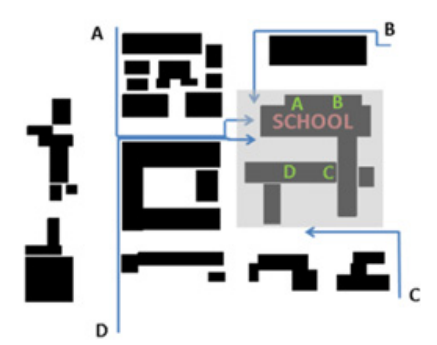

Figure 3. Domain with representation of the 4 pathways to the school (in blue) and the location of the classroom of each one (in green).

to traffic, and especially when crossing lights are present, is taken into account. Figure 3 illustrates the 4 chosen pathways within the domain and the location of the classroom each of the children attended.

\subsection{Air quality and exposure modelling}

Local scale air quality modelling was performed using the CFD model VADIS [10, 11], which has two main modules. In the first, the 3D wind flow is simulated applying a RANS prognostic model with a standard k- $\varepsilon$ turbulence closure, which has been extended for the simulation of green canopy effects [11]. In the second module, VADIS tracks the dispersion of the simulated air pollutant applying a Lagrangian approach. The needed input information on road traffic emissions was estimated using the Transport Emission Model for Line Sources (TREM) [10] based on vehicles counting and average speed.

In the core of the exposure model MEB [12] is the calculation of the individual exposure through the application of the microenvironment approach from [13]:

$$
\exp _{\mathrm{i}}=\mathrm{C}_{\mathrm{i}} \times \mathrm{t}_{\mathrm{i}}
$$


where $\exp _{i}$ is the total exposure for the person $\mathrm{i}$ over the specified period of time; $\mathrm{C} i$ is the pollutant concentration in a given location, and ti is the time spent by the person $\mathrm{i}$ in that specific location. As a result, the exposure value is expressed in concentration x time (e.g., $\mu$ g. $\mathrm{m}^{-3} . \mathrm{h}$ ), and thus can be interpreted as the mean pollutant concentration value to which the individual has been exposed during a given period of time.

To take into account exposure in indoor microenvironments (in this specific case, classrooms) the MEB model has been modified. MEB is now able to read from an input file if the individual is in an indoor or outdoor microenvironment. If the child is outdoors, it calculates exposure directly, otherwise, if the child is indoors, MEB calculates the indoor concentration from a set of pre-defined indoor/outdoor relations, and therefore, it calculates exposure. In this specific case, the indoor concentration of PM10is calculated according to equation 2 [14] that is specifically defined for indoor concentrations of PM in indoor environments during the day, based on various monitoring studies:

$$
\mathrm{C}_{\text {indoor }}=48+0.55 \times \mathrm{C}_{\text {outdoor }}
$$

The input data for MEB model is the following:

- the temporal variation of the individual location: this information is provided by the GPS equipment with predefined temporal resolution, with the information if the child is in an indoor or outdoor environment; when the child is indoors it is assumed that it is in the classroom;

- the temporal variation of the spatial distribution of concentrations at an average inhalation height. Combining, in each time-step, the georeferenced positioning of the individual with the corresponding concentration, the model tracks the time evolution of the exposure. The output data produced by MEB are both instant and mean exposure values.

\section{RESULTS AND DISCUSSION}

Air quality simulations were conducted with VADIS for a period of 31 hours, according to the time interval requirements of Directive 2008/50/CE. For more details about the modelling procedures, configurations, results and validation see [15]. Aiming to focus the analysis on a typical weekday including the morning rush hour the period of 8h00-13h00 a.m. on May $5^{\text {th }} 2004$ has been selected. This period includes the morning walk of students to the school, and the time they spend in classrooms during the morning.

\subsection{Air quality}

The simulations carried out with VADIS were validated with the measurements from the air quality station. The data comparison for the considered time period reveals a good agreement between measured and simulated values (for more details see [15]). As an example, Figure 4 presents a horizontal plane representing the hourly average of PM10 concentration for the period 8-9 a.m.

From the analysis of this particular hourly period it can be concluded that the buildings and trees on both sides of the main avenue induce a "canyon effect" on the wind flow, which leads to the formation of a hot-spot over its central axis. The maximum concentration in the domain is located over the roundabout, on the left side of the figure.

\subsection{Exposure}

In Figure 5 the evolution of the mean PM10 exposure at each time-step (every 10 seconds) is presented for the 4 walking routes considered. Notice that different routes take distinct time to reach the school. As a comparison, Figure $6 \mathrm{~b}$ shows the mean individual exposure in each route. 


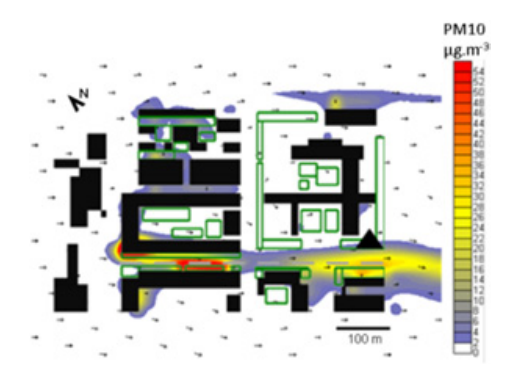

Figure 4. Horizontal PM10 concentration field for the period between 8 and 9 a.m. on May $5^{\text {th }} 2004$. Buildings are shown as black rectangles and tree blocks as unfilled green rectangles.

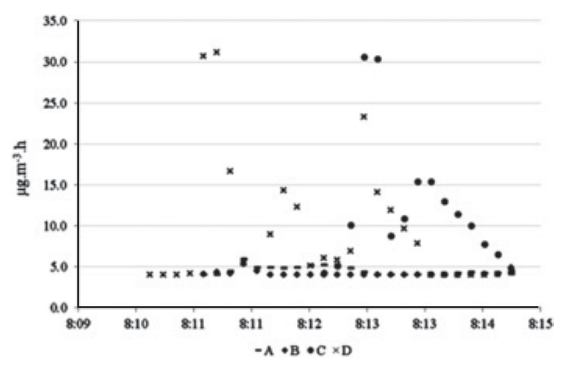

Figure 5. Time evolution of the PM10 exposure $\left(\mu \mathrm{g} \cdot \mathrm{m}^{-3}\right.$.h) for each of the 4 considered pathways.

As it can be seen from the analysis of Fig. 5, the values of the individual exposure to PM10 vary significantly, from pathway to pathway, ranging from values close to 5 to $31 \mu \mathrm{g} \cdot \mathrm{m}^{-3}$.h. Consequently, also the average exposure assumes distinct values, as shown in Figure 6b. This behaviour is a direct consequence from the asymmetric distribution of PM10 in the domain, characterised by the formation of hot-spots, as represented in Figure 4. From the point of view of health studies this fact suggests that even in a small domain a significant error can potentially occur if a mean air quality value is used as a proxy for the exposure of the individuals that use that specific microenvironment. From the modelling point of view, this conclusion shows the importance of using detailed 3D city models for this type of analysis. The accuracy and resolution of the virtual domain used by the model will ultimately affect the behaviour of the wind flow in the street-canyons, the dispersion of the emitted air pollutants and, finally, the concentration (and its distribution in the 3D space) to which individuals are exposed.When the different pathways are compared it can be concluded that children A and B have lower exposure values than $\mathrm{C}$ and $\mathrm{D}$, consistently with the air quality results that show higher accumulation of pollutant in the SW of the domain, coincident with the pathways of children C and D.

Figure 6a shows the evolution of the mean PM10 exposure at each time-step (every hour) for the 4 considered classrooms. Figure $6 \mathrm{~b}$ shows the mean individual exposure in each microenvironment (pathway and classroom) as well as total exposure.

Even using a simple approach as the indoor/outdoor ratios for determining the PM10 concentrations indoors, it can be seen that indoor spaces in the same building may have quite different concentration of pollutants, and consequently, exposure values. Again, children C, and particularly D, that attend classes in rooms in the SW of the domain, are exposed to higher values of PM10 (Figure 6a) resulting in higher indoor and total exposures (Figure 6b).

One of the limitations with these modelling approaches (for outdoor and particularly for indoor spaces) is the description of the geometry and physical characteristics of the domain of simulation. In this sense the 3D city models, appear as powerful tools for the detailed description of the geometry and properties of outdoor and indoor spaces in these studies, potentially improving human health 

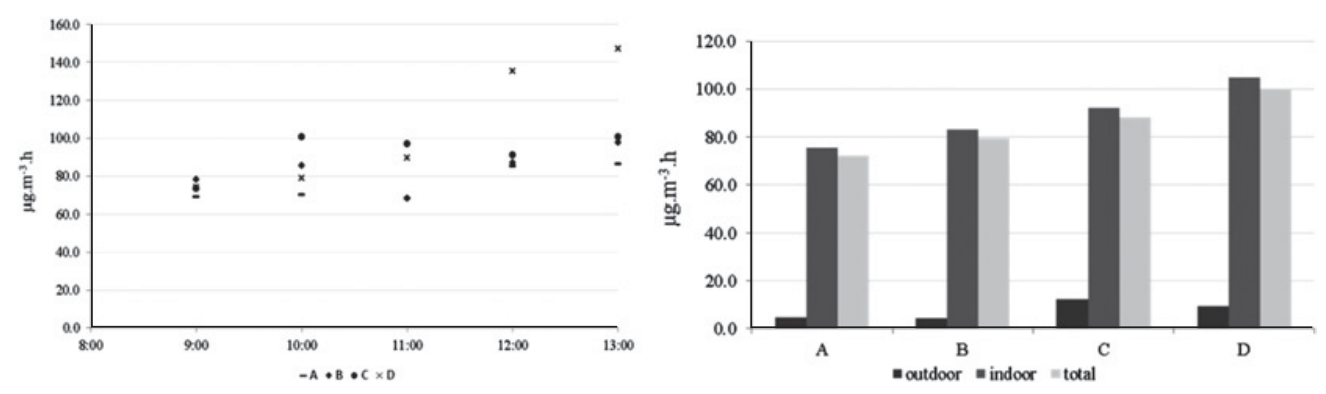

Figure 6. a) Time evolution of the PM10 exposure $\left(\mu \mathrm{g} . \mathrm{m}^{-3} . \mathrm{h}\right)$ for each of the 4 considered classrooms. b) Mean exposure values for outdoor (pathway) and indoor (classroom) microenvironments as well as total exposure values.

studies. Some authors [16] have already tested this coupling (of air quality models and 3D city models) using ontology based approaches [17] for outdoor spaces indicating clear advantages for environmental studies, as well as for urban planning studies. For instance, the thermal characteristics of a surface, important information for CFD models, can be easily derived from enriched 3D objects, that besides geometry and orientation have information about material, colour, etc. The integration of 3D city models with CFD models, used for indoor and outdoor air quality modelling, is an important step forward that can revolutionize the way that objects are included in these models. This improvement in air quality models is an important step for the air quality exposure studies, since mainly the indoor description of spaces is highly improved, being crucial for lifetime exposures and for the determination of important pollutants emitted indoors, different from the traditional regulated pollutants in the ambient air.

This study shows, through a local scale computational approach, that the individual exposure of pedestrians in an urban area is extremely spatially dependent, as a consequence of the wind flow and air pollutant dispersion patterns and characteristics. Also, their exposure in the indoors varies along with the outdoor concentration's variation, even only taking into account the outdoor concentration variation to determine the indoor one. With the increase of the detail of 3D city models, of CFD models accuracy and hardware performances, the near future will bring a deeper understanding of the relation between urban planning, quality of life and public health, as a needed step to healthier and sustainable cities.

The authors would like to acknowledge the COST Action TU 0801 "Semantic Enrichment of 3D City Models for Sustainable Urban Development", and the financial support of the 3rd European Framework Program and the Portuguese Ministry of Science, Technology and Higher Education, through the Foundation for Science and Technology (FCT), for the Post-Doc grants of J. Valente (SFRH/BPD/78933/2011), and J.H. Amorim (SFRH/BPD/48121/2008) and also projects INSPIRAR (PTDC/AAC-AMB/103895/2008) and SMARTDECISION (PTDC/SEN-TRA/115117/2009).

\section{References}

[1] EEA, Office for Official Publications of the European Communities (2005)

[2] EEA, Office for Official Publications of the European Communities (2003)

[3] Weschler, CJ; 2009. Changes in indoor pollutants since the 1950s. Atmospheric Environment. Vol. 43, n. ${ }^{\circ}$ 1, p. 153-169

[4] M. Braniš, J. Šafránek, and A. Hytychová, 2009. Build Environ 44 (86) 1246-1252 (2009)

[5] M. Stranger, S. Potgieter-Vermaak, and R. Van Grieken, Environ Int 33, 789-797 (2007)

[6] R. Goyal, and M. Khare, Atmos Environ, 43(38), 6026-6038 (2009)

[7] J.M. Daisey, W.J. Angell, and M.G. Apte, Indoor Air, 13 (1), 54-64 (2003) 
[8] D.C. Chalupa, P.E. Morrow, G. Oberdorster, M.J. Utell, and M.W. Frampton, Environ Health Persp, 112 (8), 879-82 (2004)

[9] J.G. Wilson and P. Zawar-Reza, Atmos Environ, 40 1053-1063 (2006)

[10] C. Borrego, O. Tchepel, A.M. Costa, J.H. Amorimand A.I. Miranda, Atmos Environ, 37 51975205 (2003)

[11] J.H. Amorim, V. Rodrigues, R. Tavares and C. Borrego,in City Centres. Advances in Urban Rehabilitation and Sustainability, 171-176 (2010)

[12] A.I Miranda, J.H. Amorim, V. Martins, P. Cascão, J. Valente, R. Ottmar, L.M Ribeiro, D.X. Viegasand C. Borrego C., Proc. of 3rd International Conference on Modelling, Monitoring and Management of Forest Fires (2011)

[13] O. Hertel, F.D. Leeuw, O. Raaschou-Nielsen, S.S. Jensen, D. Gee, O. Herbarth, S. Pryor, F. Palmgren and E. Olsen, Pure Appl Chem, 73 (6), 933-958 (2001)

[14] USEPA,Environmental Protection Agency (1997)

[15] V. Rodrigues, PhD Thesis, University of Aveiro (2009)

[16] C. Métral, G. Falquet, K. Karatsas, In: Teller J, Tweed C and Rabino G (eds) Conceptual Models for Urban Practitioners. Società Editrice Esculapio, 27-42 (2008)

[17] O. Corcho, M. Fernández-López, A. Gómez-Pérez, Data Knowl Eng 46 41-64 (2003) 